\title{
A Novel Switching Delayed PSO Algorithm for Estimating Unknown Parameters of Lateral Flow Immunoassay
}

\author{
Nianyin Zeng, Zidong Wang*, Hong Zhang and Fuad E. Alsaadi
}

\begin{abstract}
In this paper, the parameter identification problem of the lateral flow immunoassay (LFIA) devices is investigated via a new switching delayed particle swarm optimization (SDPSO) algorithm. By evaluating an evolutionary factor in each generation, the velocity of the particle can adaptively adjust the model according to a Markov chain in the proposed SDPSO method. During the iteration process, the SDPSO can adaptively select the inertia weight, acceleration coefficients, locally best particle pbest and globally best particle gbest in the swarm. It is worth highlighting that the pbest and the gbest can be randomly selected from the corresponding values in the previous iteration. That is, the delayed information of the pbest and the gbest can be exploited to update the particle's velocity in current iteration according to the evolutionary states. The strategy can not only improve the global search but also enhance the possibility of eventually reaching the gbest. The superiority of the proposed SDPSO is evaluated on a series of unimodal and multimodal benchmark functions. Results demonstrate that the novel SDPSO algorithm outperforms some well-known PSO algorithms in aspects of global search and efficiency of convergence. Finally, the novel SDPSO is successfully exploited to estimate the unknown time-delay parameters of a class of nonlinear state-space LFIA model.
\end{abstract}

\section{Index Terms}

Switching delayed particle swarm optimization (SDPSO); Lateral flow immunoassay; Markov chain; Timedelay; Immunochromatographic strip.

\section{INTRODUCTION}

Lateral flow immunoassay (LFIA) has been investigated extensively and has become the most widely used membrane-based immunoassay application due to its good characteristics such as remarkable usability, short assay time, good specificity and sensitiveness [13], [20]. Up to now, the LFIA has been used in

This work was supported in part by the Royal Society of the U.K., the Alexander von Humboldt Foundation of Germany, the Natural Science Foundation of China under Grant 61403319, the Fujian Natural Science Foundation under Grant 2015J05131, and the Fujian Provincial Key Laboratory of Eco-Industrial Green Technology. Asterisk indices corresponding author.

N. Zeng and H. Zhang are with the Department of Mechanical and Electrical Engineering, Xiamen University, Xiamen, Fujian 361005, P.R. China. Email address: znyexmu.edu.cn

Z. Wang is with the Department of Computer Science, Brunel University London, Uxbridge, Middlesex, UB8 3PH, United Kingdom. He is also with the Communication Systems and Networks (CSN) Research Group, Faculty of Engineering, King Abdulaziz University, Jeddah 21589, Saudi Arabia. Email address: Zidong.Wang@brunel.ac.uk

F. E. Alsaadi is with the Communication Systems and Networks (CSN) Research Group, Faculty of Engineering, King Abdulaziz University, Jeddah 21589, Saudi Arabia. 
a variety of biomedical applications including clinical diagnostics, toxins in food and agriculture products, industrial testing, and biowarfare [8], [10], [14], [37], [38]. Despite its advantages when applied to the point-of-care testing (POCT), the LFIA developed so far has been recognized to have certain weakness in quantifying test results which, to some extent, limits the scope of further applications. As such, a variety of advanced strategies based on the material selection (see e.g. [11], [15], [24]) have been developed for enhancing the biochemical characteristics of the LFIA technology over the past decade. On the other hand, there has recently been an increasing research interest in developing a dynamic model to describe the mechanism of the biochemical reactions of LFIA in order to optimize and enhance LFIA performance for the quantification, see e.g. [18], [19], [30]-[33], [35].

In this paper, we focus on the issue of modeling LFIAs, and the latest progress is given here. A mathematical model for two formats of LFIAs, namely, sandwich and competitive formats, is constructed via the convection-diffusion-reaction equations in [18], [19], and such a model has been utilized to study the performance of LFIA under various operating conditions. Different from the techniques proposed in [18], [19], an improved nonlinear state-space model has been developed in [30]-[32] for sandwich-type LFIA devices, where the process of the concentration distribution of LFIA devices is described by the nonlinear state equations and the available observation signals are modeled by the observation equation with measurement noises. It is worth highlighting that the established nonlinear state-space model provides insights into the process of the biochemical reactions of LFIA and can be utilized to test the LFIA system in an inexpensive and rapid way. Unfortunately, the signal transmission delay, which is an inherent behavior of the biochemical reactions of LFIA, has not received adequate attention in [30]-[32] despite the fact that the sample containing the target analytes does need time to flow from the sample pad along the nitrocellulose membrane encountering the detection zone via capillary action. It is, therefore, the main objective of this paper is to propose a novel approach for identifying the unknown time-delay parameters of the improved LFIA model, where dedicated efforts are devoted to the inherent time-delays among the biochemical reactions.

From the theoretical point of view, it is a challenging task to estimate unknown time-delay parameters of the improved LFIA model by using traditional optimization methods for the following three reasons. First, the available measurement is associated with a couple of specific state variables but many other state variables are simply immeasurable. Second, the established model for the LFIA devices is practically a nonlinear one and the measurement may not be directly related to the time-delays. Third, the biochemical reaction process is typically fast and the time-delays only exist at the first stage of the reaction process. In this case, it becomes extremely difficult (if not impossible) to identify the time-delays in an analytical way, and a practical alternative is to use the stochastic-search-based optimization approaches for which finding the global optima in the object optimization function is the main issues [34]. In search of a suitable optimization algorithm for modeling the LFIA, the particle swarm optimization (PSO) algorithm stands out as an appropriate candidate owing to its good performance in solving various difficult real-world optimization problems.

PSO is a stochastic-search-based optimization approach [12] that mimics the swarm behaviors of birds 
flocking or fish schooling, and has been widely exploited for solving many difficult real-world optimization problems [12], [22], [25], [26], [34]. However, an issue with PSO is that it can easily fall into the local optima when applied in the multi-modal and high-dimensional tasks, and this has restricted the PSO algorithm to wider applications [1], [25], [27], [36]. Up to now, quite a considerable number of improved PSO algorithms have been introduced to overcome this limitation, see e.g. [1], [21]-[23], [25]-[27], [34], [36]. Especially, an adaptive PSO algorithm based on an evolutionary factor has recently been introduced in [36] which can adaptively control the parameters of PSO, including the inertia weight, two acceleration coefficients and others. Furthermore, a switching PSO (SPSO) algorithm was developed in [25] where the velocity equation is determined by a Markov chain, which greatly enhance the performance of global search. Moreover, a new switching local evolutionary PSO (SLEPSO) that combined the SPSO algorithm with differential evolution has been recently developed and analyzed in [34] for quantification determination of the LFIA. Nevertheless, there is still some room to further the PSO algorithm so as to enhance its capability of getting rid of the local trapping phenomenon (premature convergence) and the introduction of time-delays with hope to enrich the dynamics could well serve this purpose.

Based on the above discussions, the main object of this study is to develop a new switching delayed PSO algorithm with delayed information and adaptive switching strategy for identifying the unknown parameters of LFIA. The proposed SDPSO algorithm can not only improve the global search but also enhance the capability of quickly reaching the gbest. The main contribution of this paper can be summarized as follows. 1) A novel switching delayed PSO (SDPSO) based on the adaptive switching strategy and the delayed information is proposed for solving the premature convergence problem, thereby improving both the search efficiency and the convergence speed of the PSO algorithm. 2) The SDPSO is verified in a comprehensive manner on a set of unimodal and multimodal benchmark functions and outperforms other six well-known PSOs. 3) The novel SDPSO method is successfully applied to identifying the unknown time-delay parameters of the improved nonlinear state-space LFIA model.

The remainder of this paper is organized as follows. The novel switching delayed PSO (SDPSO) is proposed in Section II. In Section III, simulation results and performance comparison with various existing PSO algorithms are provided. In Section IV, the improved model of LFIA device with time-delay is provided and the novel SDPSO algorithm for identifying the unknown parameters of LFIA is presented and then discussed. Finally, conclusions are drawn in Section V.

\section{A Novel Switching Delayed Particle Swarm Optimization Algorithm}

\section{A. Traditional PSO Algorithm and Its Developments}

The PSO, first introduced by Kennedy and Eberhart in 1995 [12], is a stochastic-search-based optimization approach that mimics the swarm behaviors of birds flocking or fish schooling to make the particles find the globally optimal solution.

In PSO [12], [25], [34], a swarm consisting of $S$ particles moves around at a certain speed in a $D$ dimensional search space. Each particle $i$ represented as a potential solution at the $k$ th iteration is related with two vectors, that is, the velocity vector $v_{i}(k)=\left(v_{i 1}(k), v_{i 2}(k), \cdots, v_{i D}(k)\right)$ and the position vector 
$x_{i}(k)=\left(x_{i 1}(k), x_{i 2}(k), \cdots, x_{i D}(k)\right)$. During the evolutionary process, the position of each particle will be automatically adjusted in the direction of the global optimum based on the previous experiences, one is the best position founded by itself (pbest) represented by $p_{i}=\left(p_{i 1}, p_{i 2}, \cdots, p_{i D}\right)$, and the other is the best position in the whole swarm (gbest) represented by $p_{g}=\left(p_{g 1}, p_{g 2}, \cdots, p_{g D}\right)$. The velocity and position of a particle $i$ at $(k+1)$ th iteration are described as follows:

$$
\begin{aligned}
& v_{i}(k+1)=w v_{i}(k)+c_{1} r_{1}\left(p_{i}(k)-x_{i}(k)\right)+c_{2} r_{2}\left(p_{g}(k)-x_{i}(k)\right), \\
& x_{i}(k+1)=x_{i}(k)+v_{i}(k+1),
\end{aligned}
$$

where $w$ is the inertia weight, $c_{1}$ and $c_{2}$ are acceleration coefficients called cognitive and social parameter, respectively; two random numbers $r_{1, j}$ and $r_{2, j}$, which are uniformly distributed in $[0,1]$.

The PSO described above has been gaining particular research attention and has been widely applied in solving a variety of optimization problems due to its simplicity of the concept and efficiency of the implementation. Meanwhile, much effort has been devoted to improve the searching performance of PSO. One of the common strategies is to introduce PSO with linearly time-varying inertia weight $w$ or acceleration coefficients $c_{1}, c_{2}$, as follows [21]-[23]:

$$
\begin{gathered}
w=\left(w_{1}-w_{2}\right) \times \frac{i t_{\max }-i t_{\mathrm{k}}}{i t_{\max }}+w_{2}, \\
c_{1}=\left(c_{1 f}-c_{1 i}\right) \times \frac{i t_{\max }-i t_{\mathrm{k}}}{i t_{\max }}+c_{1 i}, \\
c_{2}=\left(c_{2 f}-c_{2 i}\right) \times \frac{i t_{\max }-i t_{\mathrm{k}}}{i t_{\max }}+c_{2 i}
\end{gathered}
$$

where $i t_{\mathrm{k}}$ is the current number of iteration and $i t_{\max }$ is a maximum number of the iteration. $w_{1}$ and $w_{2}$ represent maximal and minimal values of inertia weight, respectively; $c_{1 i}$ and $c_{2 i}$ denote the minimal values, and $c_{1 f}$ and $c_{2 f}$ denote the maximum values of the acceleration coefficients $c_{1}$ and $c_{2}$, respectively.

In [22], [23], a linearly decreasing inertia weight $w$ with the iteration generations (PSO-LDIW) shown in Eq. (2) was introduced according to the characteristic of searching process. That is, the swarm inclines to perform the global exploration when the inertia weight gets larger, and the swarm tends to perform the local exploration when the inertia weight is smaller [22]. For this purpose, the maximal and minimal values are usually set as $w_{1}=0.9$ and $w_{2}=0.4$, respectively. In addition, the acceleration coefficients of the PSO are time-varying (PSO-TVAC) shown in Eq. (3)-(4) was introduced in [21] with $c_{1 i}=2.5$, $c_{2 i}=0.5, c_{1 f}=0.5$ and $c_{2 f}=2.5$. Furthermore, the constriction factor has been introduced into PSO by Clerc and Kennedy [2] to improve its search performance, where $w=0.729$ and $c_{1}=c_{2}=1.49$ were suggested in the PSO-CK algorithm. Recently, an adaptive PSO was proposed in [36] that is capable of automatically controlling the inertia weight $w$, acceleration coefficients $c_{1}, c_{2}$ and other parameters according to an evolutionary factor. Furthermore, a switching PSO algorithm was developed in [25] where the velocity equation is determined by a Markov chain, which greatly enhances the performance of global search. Moreover, a switching local evolutionary PSO (SLEPSO) that combined the SPSO algorithm with differential evolution has been recently developed and analyzed in [34] for quantification determination of the LFIA. 


\section{B. A Novel Switching Delayed PSO Algorithm}

In this section, a new switching delayed PSO (SDPSO) is proposed to improve the searching performance of PSO. The main idea of the proposed SDPSO is the velocity of the particle adaptively adjust the model according to an evolutionary factor and the Markov chain. Moreover, the delayed information of the pbest and the whole swarm gbest is exploited to update the velocity of a particle in current iteration according to the evolutionary states. This strategy can effectively prevent the PSO from the premature convergence, and this is especially useful in dealing with the multi-modal and high-dimensional problems.

1) Switching Delayed PSO Framework: The velocity and position equations of the proposed SDPSO algorithm are given as follows:

$$
\begin{aligned}
v_{i}(k+1)= & w(k) v_{i}(k)+c_{1}(\xi(k)) r_{1}\left(p_{i}\left(k-\tau_{1}(\xi(k))\right)-x_{i}(k)\right) \\
& +c_{2}(\xi(k)) r_{2}\left(p_{g}\left(k-\tau_{2}(\xi(k))\right)-x_{i}(k)\right), \\
x_{i}(k+1)= & x_{i}(k)+v_{i}(k+1)
\end{aligned}
$$

where $c_{1}(\xi(k))$ and $c_{2}(\xi(k))$ are the acceleration coefficients. The constants $\tau_{1}(\xi(k))$ and $\tau_{2}(\xi(k))$ denote the delay. All of these parameters are determined by a non-homogeneous Markov chain $\xi(k)(k \geq 0)$. The value of the Markov chain is taken in a finite state space: $\mathcal{S}=\{1,2, \cdots, N\}$. The probability transition matrix of the Markov chain is represented by $\Pi^{(k)}=\left(\pi_{i j}^{(k)}\right)_{N \times N}$, where $\pi_{i j}^{(k)} \geq 0(i, j \in \mathcal{S})$ and $\sum_{j=1}^{N} \pi_{i j}^{(k)}=1$.

In the SDPSO algorithm, the matrix $\Pi^{(k)}$ is adaptively adjusted by evaluating an evolutionary factor (EF) [25], which is firstly introduced to describe the population distribution properties in [36]. According to the characteristics of the searching process, the EF approach can be exploited to define four states: convergence, exploration, exploitation and jumping out. Especially, these four states are respectively represented by $\xi(k)=1, \xi(k)=2, \xi(k)=3$ and $\xi(k)=4$ in the Markov chain.

The average distance between each particle and the other particles in the swarm, which is represented by $d_{i}$, can be computed by,

$$
d_{i}=\frac{1}{S} \sum_{i=1}^{S} \sqrt{\sum_{k=1}^{D}\left(x_{i}^{k}-x_{j}^{k}\right)^{2}}
$$

where $S$ and $D$ stand for the swarm size and the dimensions, respectively. Hence, the evolution factor $E_{f}$ can be defined as follows [36]:

$$
E_{f}=\frac{d_{g}-d_{\min }}{d_{\max }-d_{\min }}
$$

where $d_{g}$ denotes the globally best particle among $d_{i} . d_{\max }$ and $d_{\min }$ denote the maximum and minimum distances in $d_{i}$, respectively.

Based on the value of evolutionary factor $E_{f}$, we can get the value of the Markov chain as follows 
TABLE I

STRATEGIES FOR SELECTING $c_{1}$ AND $c_{2}$

\begin{tabular}{|c|c|c|c|}
\hline \hline State & Mode & $c_{1}$ & $c_{2}$ \\
\hline Convergence & $\xi(k)=1$ & 2 & 2 \\
\hline Exploitation & $\xi(k)=2$ & 2.1 & 1.9 \\
\hline Exploration & $\xi(k)=3$ & 2.2 & 1.8 \\
\hline Jumping-out & $\xi(k)=4$ & 1.8 & 2.2 \\
\hline \hline
\end{tabular}

[25]:

$$
\xi(k)=\left\{\begin{array}{cc}
1, & 0 \leq E_{f}<0.25 \\
2, & 0.25 \leq E_{f}<0.5 \\
3, & 0.5 \leq E_{f}<0.75 \\
4, & 0.75 \leq E_{f}<1
\end{array}\right.
$$

where the probability transition matrix is provided by:

$$
\Pi=\left(\begin{array}{cccc}
\chi & 1-\chi & 0 & 0 \\
\frac{1-\chi}{2} & \chi & \frac{1-\chi}{2} & 0 \\
0 & \frac{1-\chi}{2} & 0 & \frac{1-\chi}{2} \\
0 & 0 & 1-\chi & \chi
\end{array}\right)
$$

Hence, the Markov process at the next iteration may switch its state based on the probability distribution matrix $\Pi$. It should be pointed out that the value of the probability $\chi$ is of great importance to keep the classification accuracy and also the search diversity $(\chi=0.9$ in this paper) [25].

2) Switching Delayed PSO Parameters: The strategies introduced in [25] for controlling the inertia weight $w$ and also selecting the acceleration coefficients $\left(c_{1}\right.$ and $\left.c_{2}\right)$ are exploited in this paper.

The inertia weight $w$ has the same tendency as the evolutionary factor $E_{f}$ during the iteration process. Note that, a large $w$ will tend to the global search in the states of jumping-out and exploration. In contrast, a small $w$ will benefit the local search. The initial value of $w$ is set as 0.9 , and the function for describing inertia weight $w$ and $E_{f}$ can be represented as follows [25]:

$$
w\left(E_{f}\right)=0.5 E_{f}+0.4 \in[0.4,0.9], \forall E_{f} \in[0,1] .
$$

On the other hand, the initial values of acceleration coefficients $c_{1}$ and $c_{2}$ are set as 2 , and both of them can automatically adjust their values based on the evolutionary state [25], which given in the Table I.

3) Strategies for Selecting Delayed Information: The part constitutes the main novelty of the proposed SDPSO algorithm where the delayed information of pbest and gbest are utilized to update the velocity equation based on the evolutionary state. The strategies for selecting delayed information are introduced as below:

- In a jumping-out state, the current globally best particle is willing to fly to a better optimum so as to escape from the local optimum. The delayed information of the pbest and gbest distribute much wider 
TABLE II

STRATEGIES FOR SELECTING DELAYED INFORMATION AND PARAMETERS

\begin{tabular}{|c|c|c|c|c|c|}
\hline \hline State & Mode & pbest & gbest & $\tau_{1}(\xi(k))$ & $\tau_{2}(\xi(k))$ \\
\hline Convergence & $\xi(k)=1$ & $p_{i}(k)$ & $p_{g}(k)$ & 0 & 0 \\
\hline Exploitation & $\xi(k)=2$ & $p_{i}\left(k-\tau_{1}(\xi(k))\right)$ & $p_{g}(k)$ & $\left\lfloor k \cdot r a n d_{1}\right\rfloor$ & 0 \\
\hline Exploration & $\xi(k)=3$ & $p_{i}(k)$ & $p_{g}\left(k-\tau_{2}(\xi(k))\right)$ & 0 & $\left\lfloor k \cdot r_{a n d}\right\rfloor$ \\
\hline Jumping-out & $\xi(k)=4$ & $p_{i}\left(k-\tau_{1}(\xi(k))\right)$ & $p_{g}\left(k-\tau_{2}(\xi(k))\right)$ & $\left\lfloor k \cdot\right.$ rand $\left._{1}\right\rfloor$ & $\left\lfloor k \cdot\right.$ rand $\left._{2}\right\rfloor$ \\
\hline \hline
\end{tabular}

in the search space. More importantly, $p_{i}\left(k-\tau_{1}(\xi(k))\right)$ and $p_{g}\left(k-\tau_{2}(\xi(k))\right.$ are the particle and the swarm encountered in the previous iteration, that is, both of them contain the knowledge/experiences of the particle and swarm. Therefore, they are selected, respectively, for updating the influences of "self-cognition" and "social" in the velocity equation, and this will help to jump out the local optimum.

- In an exploration state, it is crucial for exploring as many optima as possible. Hence, selecting the pbest at the current iteration and the delayed value of gbest can make particles explore individually and also lead particles to fly to historically global best positions (instead gathering around the current global best position which could possibly be a local optimum).

- In an exploitation state, each particle is taking advantage of its historical best position $p_{i}\left(k-\tau_{1}(\xi(k))\right)$ and gbest at the current iteration to enhance the search and exploitation around the local region.

- In the convergence state, all particles are willing to converge to the best optima as soon as possible in the found globally optimal region. Hence, the particles should follow the pbest and gbest at the current iteration to achieve this goal in this state.

The strategies discussed above can be summarized in Table II. It should be mentioned that the constants $\tau_{1}(\xi(k))$ and $\tau_{2}(\xi(k))$ are selected randomly, which are given in Table II. $\lfloor\cdot\rfloor$ is the floor function. In other words, the pbest and the gbest are selected randomly from the values encountered in the previous iteration.

The flowchart of the proposed Switching delayed PSO (SDPSO) is illustrated in Fig.1.

\section{Simulation EXPERIMENTS}

\section{A. The Benchmark Functions}

In the experiments, some well-known benchmark functions with different properties given by (11)-(15) are utilized to demonstrate the performance of the new SDPSO approach. Especially, these benchmark functions have their own characteristics. The Sphere function $f_{1}(x)$ is generally exploited to evaluate the convergent rate of the algorithm since $f_{1}(x)$ is a typical unimodal optimization problem. $f_{2}(x)$ is very difficult to optimize since the optimum locates in a banana-shaped valley and therefore can be seen as a multimodal problems. Besides, there is no doubt it is hard to optimize $f_{3}(x)$ and $f_{5}(x)$ functions which are typical multimodal problems. 


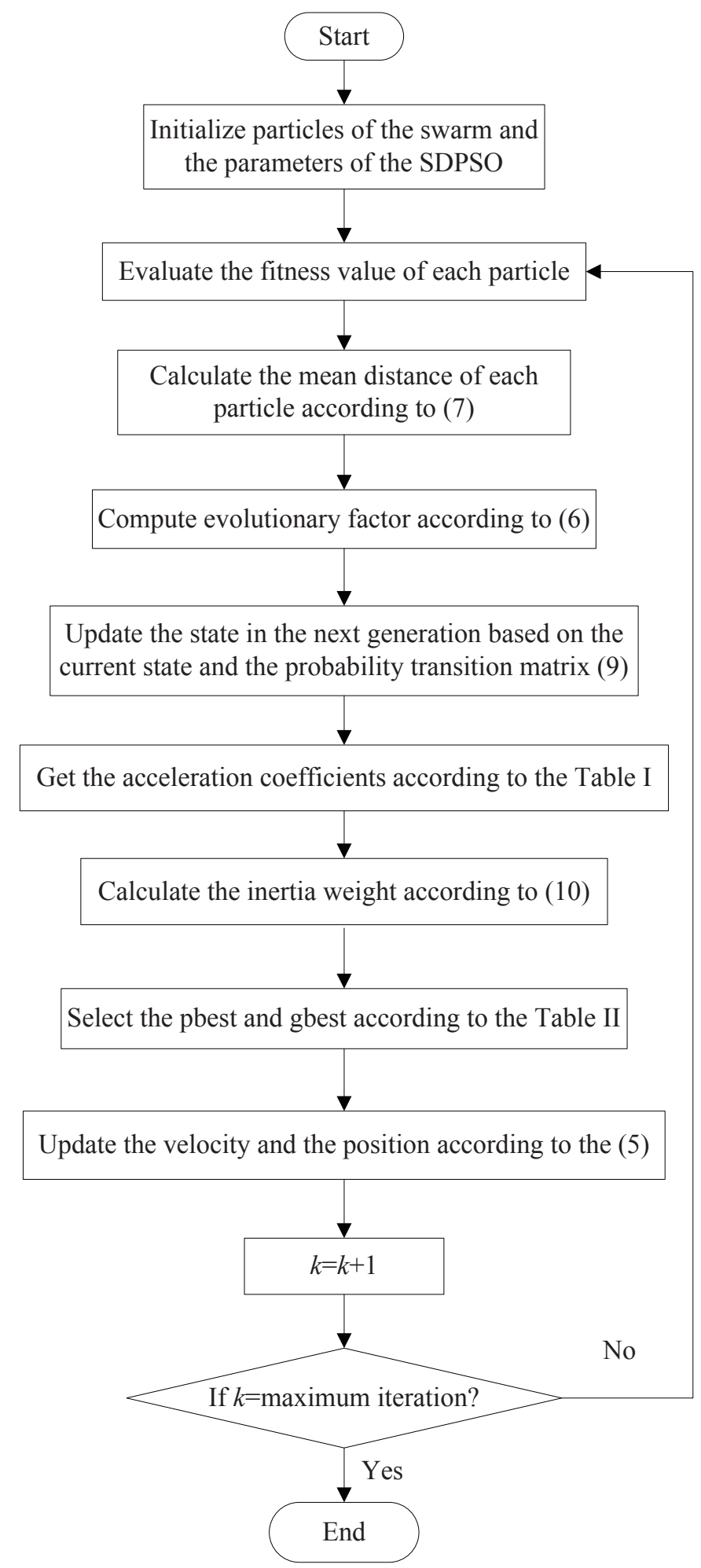

Fig. 1. The flowchart of SDPSO algorithm

$$
\text { Sphere : } f_{1}(x)=\sum_{i=1}^{D} x_{i}^{2} \text {, and } x \in[-100,100]^{D} \text {. }
$$




$$
\begin{gathered}
\text { Rosenbrock : } f_{2}(x)=\sum_{i=1}^{D-1}\left(100\left(x_{i+1}-x_{i}\right)^{2}+\left(x_{i}-1\right)^{2}\right) \text {, and } x \in[-10,10]^{D} \text {. } \\
\text { Ackley : } f_{3}(x)=-20 e^{-0.2 \sqrt{\frac{1}{D} \sum_{i=1}^{D} x_{i}^{2}}}-e^{\frac{1}{D} \sum_{i=1}^{D} \cos 2 \pi x_{i}}+20+e, \text { and } x \in[-32,32]^{D} \text {. } \\
\text { Griewank : } f_{4}(x)=\frac{1}{4000} \sum_{i=1}^{D} x_{i}^{2}-\prod_{i=1}^{D} \cos \frac{x_{i}}{\sqrt{i}}+1, \text { and } x \in[-600,600]^{D} . \\
\text { Rastrigin : } f_{5}(x)=\sum_{i=1}^{D}\left(x_{i}^{2}-10 \cos 2 \pi x_{i}+10\right) \text {, and } x \in[-50,50]^{D} .
\end{gathered}
$$

\section{B. Simulation Results and Discussion}

The experiments are conducted to verify the effectiveness of the novel SDPSO algorithm and compare the SDPSO with six well-known PSOs to demonstrate its superiority. The parameters of these five benchmark functions in the experiments are set as: the particle number $N=20$, the dimension $D=20$, the maximum generation number is 3000 . Especially, each experiment of the same algorithm is repeated 20 times independently so as to eliminate the random effect, and then the mean result is provided. Moreover, we compare the proposed SDPSO algorithm with six other well-known PSO algorithms, which consist of the PSO-LDIW [22], [23], PSO-TVAC [21], PSO-CK [2], simplified SPSO [25], PSO combing with differential evolutionary algorithm (LEPSO) [1] and a hybrid SPSO with the differential evolutionary algorithm SLEPSO [34]. The parameters for these PSOs can be seen in [34] for more details.

As shown in the Table III and Figs. 2-6, the proposed SDPSO algorithm outperforms six other PSO algorithms in term of a series of criteria. Particularly, the best result among those PSOs for each benchmark function is highlighted by boldface in the table. Obviously, the SDPSO method could find an optimal solution with high accuracy and fast speed. The Sphere function $f_{1}(x)$ is generally exploited to evaluate the convergent rate of the algorithm. Therefore, we can conclude that the local search ability of the new SDPSO method is better than other six well-known PSOs from Table III and Fig. 2. The Rosenbrock function $f_{2}(x)$, which is viewed as a mono-modal optimization problem is often used to verify not only the local search but also the global search abilities. It can be seen from Table III and Fig. 3 that SDPSO performs better than the other six PSO algorithms. The other three benchmark functions are generally utilized to verify the global search ability of the PSO approach. From Table III and Figs. 4-6, it is clear that the new SDPSO approach outperforms the other six algorithms. Hence, the SDPSO method proposed in this paper performs best among seven PSOs on both unimodal and multimodal functions.

Remark 1: Note that the SDPSO method is capable of solving the optimization problem with fast convergence as well as global search accuracy characteristics due to the use of the adaptive switching strategy and the delayed information embedded in algorithm. Moreover, the advantage of escaping from local optima and converging to global optimum of multimodal functions shows the superiority of the proposed SDPSO algorithm. 
TABLE III

THE COMPARISONS OF SEARCH RESULTS AMONG SEVEN PSOS ON FIVE BENCHMARK FUNCTIONS

\begin{tabular}{|c|c|c|c|c|c|c|c|c|}
\hline & & PSO-TVAC & PSO-CK & PSO-LDIW & LEPSO & SPSO & SLEPSO & SDPSO \\
\hline \multirow[t]{3}{*}{$\overline{f_{1}}$} & Best value & $9.25 \times e^{-38}$ & $5.89 \times e^{-71}$ & $2.73 \times e^{-32}$ & $1.24 \times e^{-30}$ & $2.85 \times e^{-70}$ & $3.19 \times e^{-69}$ & $\mathbf{0}$ \\
\hline & Mean & $1.12 \times e^{-18}$ & $1.66 \times e^{-57}$ & $3.14 \times e^{-29}$ & $5.23 \times e^{-28}$ & $7.97 \times e^{-66}$ & $4.58 \times e^{-65}$ & $\mathbf{0}$ \\
\hline & Std. Dev. & $4.98 \times e^{-18}$ & $7.4 \times e^{-57}$ & $6.94 \times e^{-29}$ & $8.76 \times e^{-28}$ & $2.32 \times e^{-65}$ & $1.07 \times e^{-64}$ & $\mathbf{0}$ \\
\hline \multirow[t]{3}{*}{$f_{2}$} & Best value & 0.027 & 0.0056 & 3.06 & 0.19 & 0.80 & 0.38 & $2.7108 \times \mathrm{e}^{-4}$ \\
\hline & Mean & 13.43 & 8.39 & 23.16 & 19.86 & 11.66 & 1.17 & 0.3072 \\
\hline & Std. Dev. & 13.87 & 14.56 & 25.40 & 21.84 & 13.75 & 0.68 & 0.4739 \\
\hline \multirow[t]{3}{*}{$f_{3}$} & Best value & $1.11 \times e^{-13}$ & 19.1803 & $7.99 \times e^{-15}$ & $7.99 \times e^{-15}$ & $4.44 \times e^{-15}$ & $4.44 \times e^{-15}$ & $8.8818 \times \mathrm{e}^{-16}$ \\
\hline & Mean & 2.2433 & 19.7191 & 3.0048 & $2.63 \times e^{-13}$ & 2.02 & $6.75 \times e^{-15}$ & $8.8818 \times \mathrm{e}^{-16}$ \\
\hline & Std. Dev. & 5.9376 & 0.1883 & 7.3383 & $3.86 \times e^{-13}$ & 6.23 & $1.74 \times e^{-15}$ & $\mathbf{0}$ \\
\hline \multirow[t]{3}{*}{$f_{4}$} & Best value & 121.6087 & 0.787 & 0 & 0 & 0 & 0 & $\mathbf{0}$ \\
\hline & Mean & 175.3641 & 29.5584 & 0.018 & 0.0047 & 0.0142 & 0 & 0 \\
\hline & Std. Dev. & 27.8352 & 20.7047 & 0.0131 & 0.0072 & 0.0128 & 0 & $\mathbf{0}$ \\
\hline \multirow[t]{3}{*}{$f_{5}$} & Best value & 22.8841 & 65.6671 & 15.9193 & 6.9647 & 13.9294 & 2.9849 & $\mathbf{0}$ \\
\hline & Mean & 39.1516 & 133.2741 & 23.5821 & 15.626 & 24.1834 & 6.5785 & $\mathbf{0}$ \\
\hline & Std. Dev. & 9.6101 & 46.8896 & 6.4960 & 5.4027 & 8.1526 & 3.7855 & $\mathbf{0}$ \\
\hline
\end{tabular}

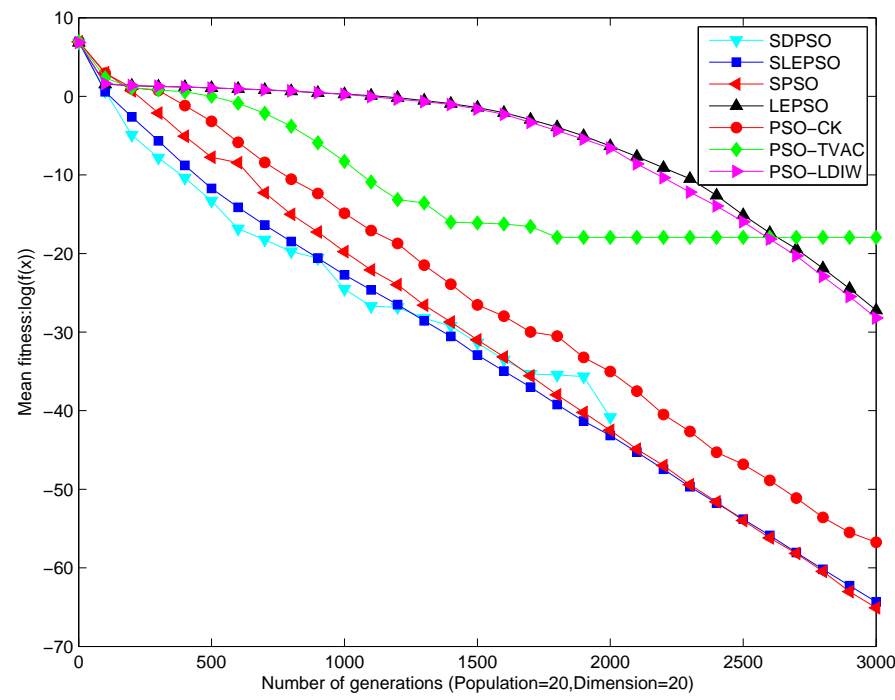

Fig. 2. The performance of seven PSOs for 20-dimensional $f_{1}(x)$.

\section{Parameter Estimation of The Improved LFIA Model}

In this section, the novel SDPSO method is utilized to estimate the unknown time-delay parameters of the improved LFIA model, where the time-delays occurring among the biochemical reactions are considered.

\section{A. The Improved Nonlinear LFIA Model}

A nonlinear state-space model has recently been developed in [30]-[32] for sandwich-type LFIA devices. The developed model includes the equations for describing the biochemical reaction process of LFIA 


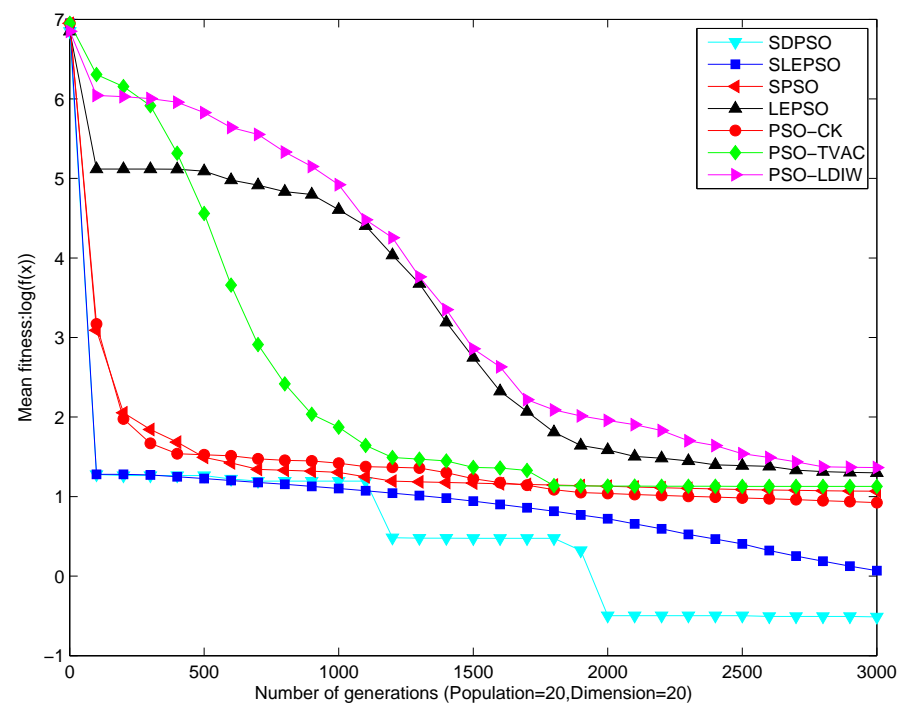

Fig. 3. The performance of seven PSOs for 20-dimensional $f_{2}(x)$.

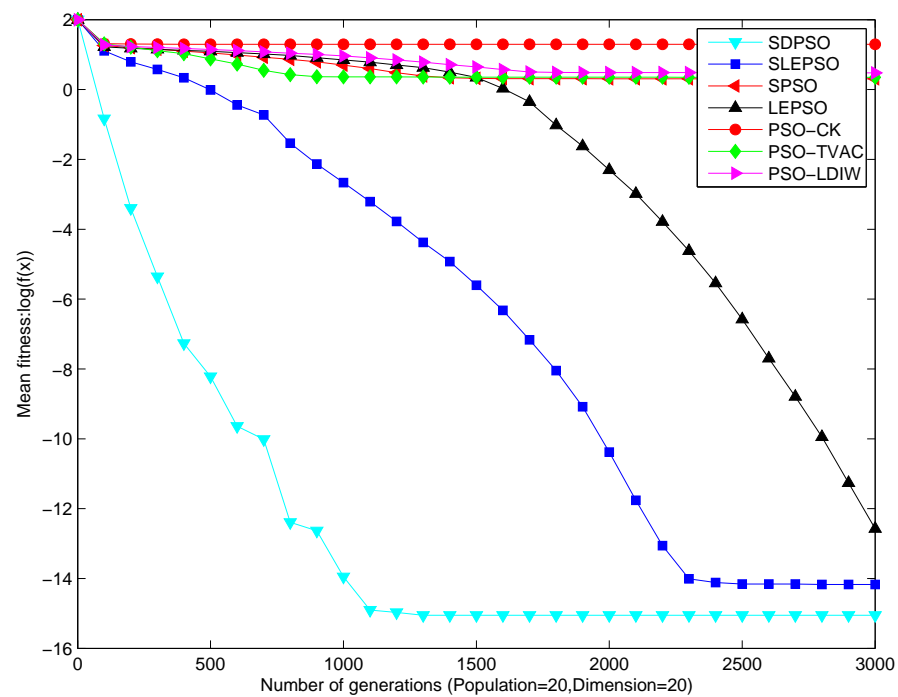

Fig. 4. The performance of seven PSOs for 20-dimensional $f_{3}(x)$.

system and the observation output. Especially, the process of the concentration distribution of LFIA devices are described by the nonlinear state equations, and the available measurement signals are characterized by the observation equation including the measurement noises. In [30]-[32], however, the time-delay has not been taken into consideration in the process of LFIA reactions. Such an assumption is not really reasonable since the sample containing the target analytes needs time to flow from the sample pad along the nitrocellulose membrane encountering the detection zone via capillary action. Hence, the time-delays between the biochemical reactions are considered in this paper. The reaction rates of the LFIA systems 


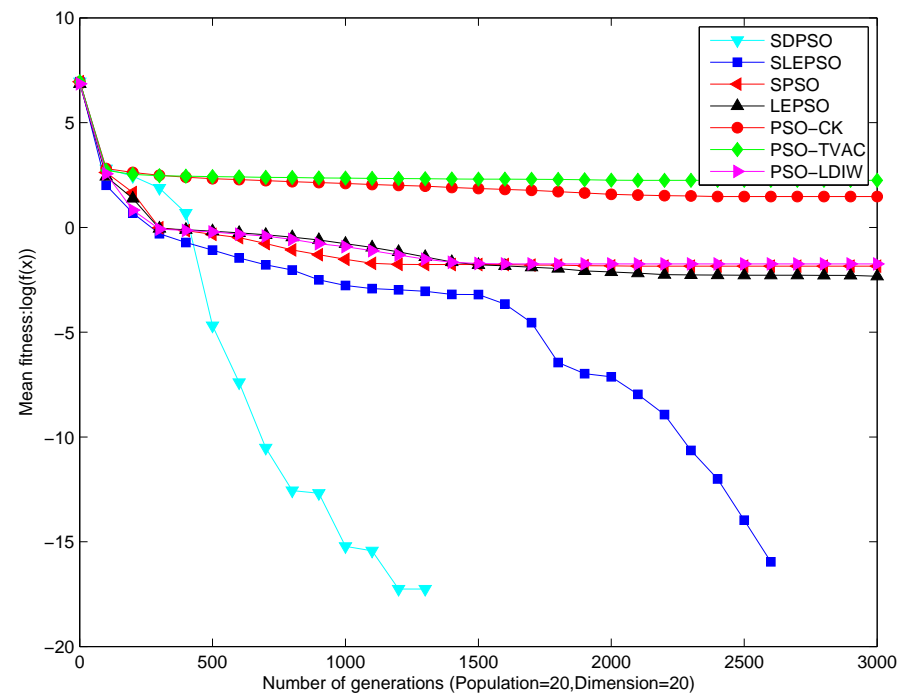

Fig. 5. The performance of seven PSOs for 20-dimensional $f_{4}(x)$.

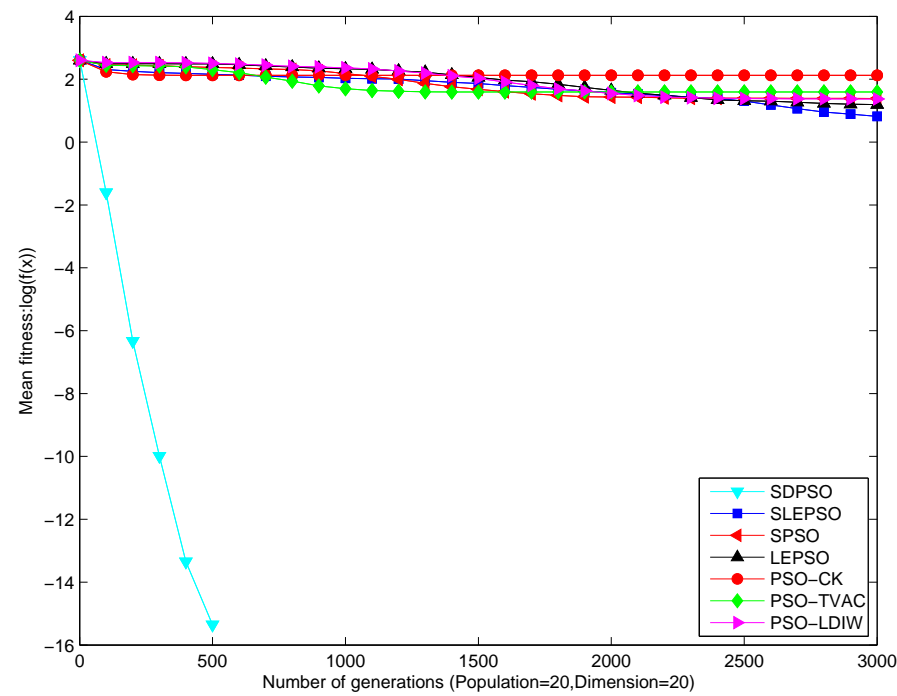

Fig. 6. The performance of seven PSOs for 20-dimensional $f_{5}(x)$.

are described as follows:

$$
\begin{aligned}
& v_{1}=k_{1} x_{1} x_{2}-k_{2} x_{3} \\
& v_{2}=\left(k_{3} x_{1} x_{4}-k_{4} x_{5}\right)\left(t-\tau_{1}\right) \\
& v_{3}=\left(k_{5} x_{3} x_{4}-k_{6} x_{6}\right)\left(t-\tau_{2}\right) \\
& v_{4}=\left(k_{7} x_{2} x_{5}-k_{8} x_{6}\right)\left(t-\tau_{3}\right)
\end{aligned}
$$

where $k_{1}, k_{3}, k_{5}, k_{7}$ and $k_{2}, k_{4}, k_{6}, k_{8}$ are, respectively, the association and dissociation rate constants, and $\tau_{1}, \tau_{2}, \tau_{3}$ are time delays which exist among the biochemical reactions. The stoichiometrix $S$ for the 
reaction process of the LFIA system is provided as follows:

$$
S=\left[\begin{array}{cccc}
-1 & -1 & 0 & 0 \\
-1 & 0 & 0 & -1 \\
1 & 0 & -1 & 0 \\
0 & -1 & -1 & 0 \\
0 & 1 & 0 & -1 \\
0 & 0 & 1 & 1
\end{array}\right] .
$$

Therefore, the improved nonlinear state-space model with time-delay of the LFIA device is shown as follows:

$$
\begin{aligned}
x(k+1) & =x(k)+S V(x(k), \tau)+w(k) \\
y(k) & =g(x(k))+v(k)
\end{aligned}
$$

where $x(k)$ denotes the vector of various materials in the LFIA device; $y(k)$ is the observation experiment value at time point $k . V=\left[v_{1}, v_{2}, \ldots, v_{4}\right]^{T}$ is the reaction rate vector. $w(k)$ and $v(k)$ stand for zero-mean uncorrelated Gaussian white noises, $w(k) \sim \mathcal{N}(0, Q)$ and $v(k) \sim \mathcal{N}(0, R)$, respectively.

\section{B. Parameter Identification and Results}

The main object of this study is to identify the unknown time-delay parameters of Eq. (20) via the proposed SDPSO algorithm. Here, the structure of the improved nonlinear model of LFIA device (20)-(21) is supposed to be known in this study. Meanwhile, the time-delay parameters $\tau_{1}, \tau_{2}, \tau_{3}$ are all viewed as unknown parameters to be estimated. Therefore, the problem of parameter estimation of the improved LFIA model can be transformed into an optimization problem, and the objective function is given by:

$$
J=100 \sum_{c=1}^{l} \sum_{k=1}^{s}\left\|z_{c k}-\hat{y}_{c k}\right\|,
$$

where $l$ denotes the dimension of observations in the state-space model, especially $l=1$ in the improved nonlinear LFIA model; $s$ is the length of observations, and $z_{c k}$ represent the actual value for the $c$ th observation at the $k$ th time point.

It should be pointed out that it is a challenging task to estimate unknown time-delay parameters of the improved LFIA model by using traditional optimization methods for the following three reasons. First, the available measurement is associated with a couple of specific state variables but many other state variables are simply immeasurable. Second, the established model for the LFIA devices is practically a nonlinear one and the measurement may not be directly related to the time-delays. Third, the biochemical reaction process is typically fast and the time-delays only exist at the first stage of the reaction process. In this case, the object optimization function (22) would have local optimums. Considering the biochemical reactions of the LFIA, we assume that the time-delay $\tau_{2}$ equals to the time-delay $\tau_{3}$, and is larger than the $\tau_{1}$. The association and dissociation rate constants of the LFIA model, which have been identified in [32], are utilized in this paper. Especially, we carried out three experiments where the concentrations of 
samples are different to comprehensively evaluate the presented SDPSO algorithm. In experiments, we set the population size as 10 and the maximum generation as 100. Meanwhile, each experiment is carried out 10 times independently so as to eliminate the random effect. Three convergence processes of the objective function $J$ are shown in Fig. 7 and the evolving processes of the identified time-delay parameters $\tau_{1}$ and $\tau_{2}$ are illustrated respectively in Fig. 8 and Fig. 9.

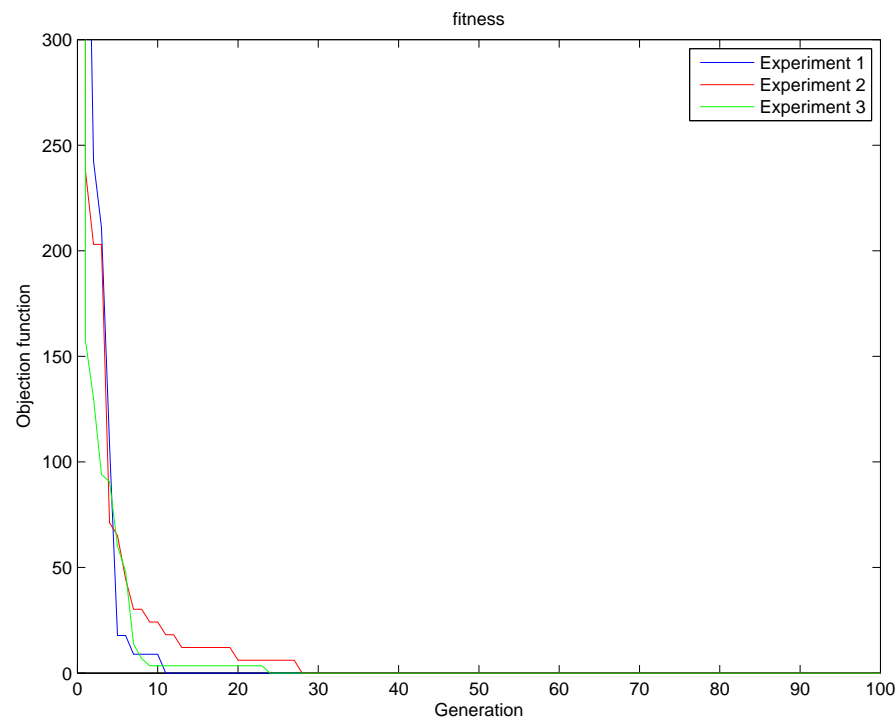

Fig. 7. Three convergence processes of the objective function $J$.

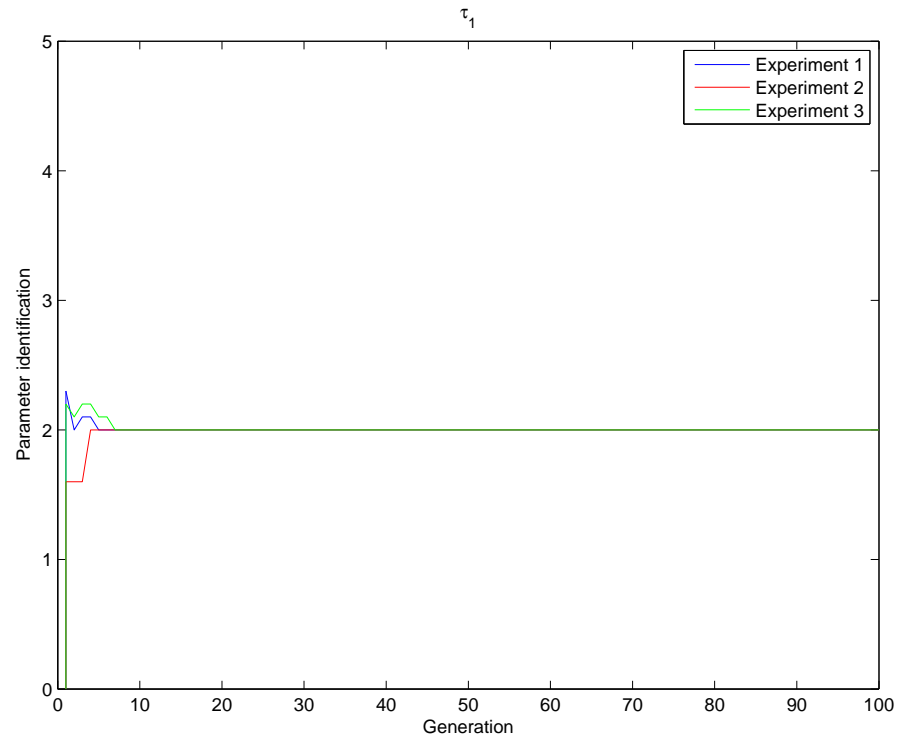

Fig. 8. Three evolving processes of time-delay parameter $\tau_{1}$.

Fig. 7 demonstrates that the values of object optimization function (22) for three experiments descend quickly to zero, which indicates that the novel SDPSO algorithm can efficiently find the global optimum. Moreover, it is obvious from Fig. 8 and Fig. 9 that time-delay parameters $\tau_{1}$ and $\tau_{2}$ for three experiments 


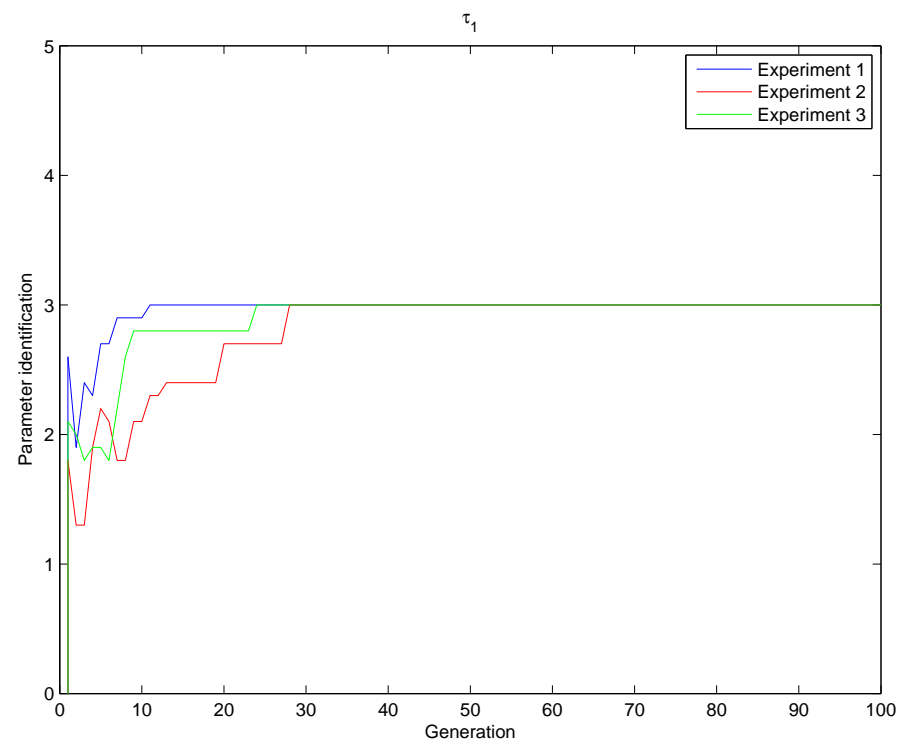

Fig. 9. Three evolving processes of time-delay parameters $\tau_{2}$.

converge to the same results quickly, which verified the high efficiency of the new SDPSO algorithm.

\section{CONCLUSIONS}

In this paper, we have developed a new switching delayed particle swarm optimization (SDPSO) algorithm for inferring the unknown time-delay parameters of the LFIA system. The velocity of the particle can adaptively adjust the mode according to a Markov chain and an evolutionary factor in the proposed SDPSO method. Therefore, the SDPSO can adaptively select the inertia weight, acceleration coefficients, itself local best particle pbest and the global best particle in the swarm gbest during the iteration process. It should be mentioned that the delayed information of the pbest and the gbest can be randomly utilized to update the particle's velocity in current iteration according to the evolutionary states. Compared with six other well-known PSOs, the search performance including the global optimality and convergence speed is improved substantially when testing a set of unimodal and multimodal benchmark functions. Finally, we have exploited the new SDPSO to identify the unknown time-delay parameters of the improved nonlinear state-space LFIA model with time-delay.

In the near future, our research topics will focus on the strategy for selecting more complicated dynamical behaviors (e.g. delayed information [6], [7], [16], [17], [29] and jumped/switched information [3]-[5], [9], [28]) so as to further improve the performance of PSO, and also on the issue of modeling the LFIA system together with the control line to comprehensively simulate the biochemical reaction process.

\section{Compliance with Ethical Standards}

Conflict of Interest Nianyin Zeng, Zidong Wang, Hong Zhang and Fuad E. Alsaadi declare that they have no conflict of interest.

Informed Consent All procedures followed were in accordance with the ethical standards of the responsible committee on human experimentation (institutional and national) and with the Helsinki Declaration 
of 1975, as revised in 2008 (5). Additional informed consent was obtained from all patients for which identifying information is included in this article.

Human and Animal Rights This article does not contain any studies with human or animal subjects performed by the any of the authors.

\section{REFERENCES}

[1] A. Abdullah, S. Deris, S. Hashim, M. Mohamad, and S. Arjunan, An improved local best searching in particle swarm optimization using differential evolution, 11th International Conference on Hybrid Intelligent Systems, pp. 115-120, 2011.

[2] M. Clerc, and J. Kennedy, The particle swarm: Explosion, stability, and convergence in a multi-dimensional complex space, IEEE Transactions on Evolutionary Computation, vol. 6, no. 1, pp. 58-73, 2002.

[3] D. Ding, Z. Wang, B. Shen and H. Dong, Event-triggered distributed $H_{\infty}$ state estimation with packet dropouts through sensor networks, IET Control Theory \& Applications, vol. 9, no. 13, pp. 1948-1955, 2015.

[4] D. Ding, Z. Wang, F. E. Alsaadi and B. Shen, Receding horizon filtering for a class of discrete time-varying nonlinear systems with multiple missing measurements, International Journal of General Systems, vol. 44, no. 2, pp. 198-211, 2015.

[5] D. Ding, Z. Wang, B. Shen and G. Wei, Event-triggered consensus control for discrete-time stochastic multi-agent systems: the inputto-state stability in probability, Automatica, vol. 62, pp. 284-291, 2015.

[6] D. Ding, Z. Wang, J. Lam and B. Shen, Finite-Horizon $H_{\infty}$ control for discrete time-varying systems with randomly occurring nonlinearities and fading measurements, IEEE Transactions on Automatic Control, vol. 60, no. 9, pp. 2488-2493, 2016.

[7] D. Ding, Z. Wang, B. Shen and H. Dong, $H_{\infty}$ state estimation with fading measurements, randomly varying nonlinearities and probabilistic distributed delays, International Journal of Robust and Nonlinear Control, vol. 25, no. 13, pp. $2180-2195,2015$.

[8] J. Gillespie, G. Gannot, M. Tangrea, M. Ahram, C. Best, V. Bichsel, E. Petricoin, M. Emmert-Buck and R. Chuaqui, Molecular profiling of cancer, Toxicol. Pathol, vol. 32, pp. 67-71, 2004.

[9] N. Hou, H. Dong, Z. Wang, W. Ren and F. E. Alsaadi, Non-fragile state estimation for discrete Markovian jumping neural networks, Neurocomputing, vol. 179, pp. 238-245, 2016.

[10] S. Huang, H. Wei and Y. Lee, One-step immunochro-matographic assay for the detection of Staphylococcus aureus, Food Control, vol. 18, no. 8, pp. 893-897, 2007.

[11] J. Kaur, K. Singh, R. Boro, K. Thampi, M. Raje and G. Varshney, Immunochromatographic dipstick assay format using gold nanoparticles labeled protein-hapten conjugate for the detection of atrazine, Environmental Science and Technology, vol. 41, no. 14, pp. 5028-5036, 2007.

[12] J. Kennedy and R. Eberhart, Particle swarm optimization, Proc. IEEE International Conference On Neural Network, pp. 1942-1948, 1995.

[13] E. Laderman, E. Whitworth, E. Dumaual, M. Jones, A. Hudak, W. Hogrefe, J. Carney and J. Groen, Rapid, sensitive, and specific lateral-flow immunochromatographic point-of-care device for detection of herpes simplex virus type 2-specific immunoglobulin $\mathrm{G}$ antibodies in serum and whole blood, Clin. Vaccine Immunol, vol. 5, pp. 159-163, 2008.

[14] R. Lundblad and P. Wagner, The potential of proteomics in developing diagnostics, IVD Technology, vol. 3, pp. 20-22, 2005.

[15] D. Li, S. Wei, H. Yang, Y. Li and A. Deng, A sensitive immunochromatographic assay using colloidal gold-antibody probe for rapid detection of pharmaceutical indomethacin in water samples, Biosensors and Bioelectronics, vol. 24, no. 7, pp. $2277-2280,2009$.

[16] Y. Liu, F. E. Alsaadi, X. Yin and Y. Wang, Robust $H_{\infty}$ filtering for discrete nonlinear delayed stochastic systems with missing measurements and randomly occurring nonlinearities, International Journal of General Systems, vol. 44, no. 2, pp. 169-181, 2015.

[17] Y. Luo, G. Wei, Y. Liu and X. Ding, Reliable $H_{\infty}$ state estimation for 2-D discrete systems with infinite distributed delays and incomplete observations, International Journal of General Systems, vol. 44, no. 2, pp. 155-168, 2015.

[18] S. Qian and H. Haim, A mathematical model of lateral flow bioreactions applied to sandwich assays, Analytical Biochemistry, vol. 322, no. 1, pp. 89-98, 2003.

[19] S. Qian and H. Haim, Analysis of lateral flow biodetectors: competitive format, Analytical Biochemistry, vol. 326, no. 2, pp. 211-224, 2004.

[20] C. Raphael and Y. Harley, Lateral flow immunoassay, Humana Press, 2008.

[21] A. Ratnaweera, SK. Halgamure, HC. Watson. Self-organizing hierarchical particle swarm optimizer with time-varying acceleration coefficients, IEEE Transactions on Evolutionary Computation, vol.8, pp. 240-55, 2004. 
[22] Y. Shi, R. C. Eberhart, Empirical study of particle swarm optimization, Proceedings of the 1999 IEEE Congress on Evolutionary Computation, pp. 1945-1950, 1999.

[23] Y. Shi, R. C. Eberhart, Parameter selection in particle swarm optimization. Proceedings of the 7th International Conference on Evolutionary Programming, pp. 591-600, 1998

[24] R. Tanaka, T. Yuhi, N. Nagatani, T. Endo, K. Kerman and Y. Takamura, A novel enhancement assay for immunochromatographic test strips using gold nanoparticles, Anal Bioanal Chem, vol. 385, no. 8, pp. 1414-1420, 2006.

[25] Y. Tang, Z. Wang and J. Fang, Parameters identification of unknown delayed genetic regulatory networks by a switching particle swarm optimization algorithm, Expert Systems with Applications, vol. 38, pp. 2523-2535, 2011.

[26] Y.G. Tang, X. Guan, Parameter estimation for time-delay chaotic system by particle swarm optimization, Chaos, Solitons and Fractals, vol. 40, no. 3, pp. 1391-1398, 2009.

[27] Y. Valle, G. Venayagamoorthy, S. Mohagheghi, J. Hernandez, and R. Harley, Particle swarm optimization: basic concepts, variants and applications in power systems, IEEE Transactions on Evolutionary Computation, vol. 12, no. 2, pp. 171-195, 2008.

[28] H. Yang, Z. Wang, H. Shu, F. E. Alsaadi and T. Hayat, Almost sure $H_{\infty}$ sliding mode control for nonlinear stochastic systems with Markovian switching and time-delays, Neurocomputing, vol. 175, pp. 392-400, 2016.

[29] Y. Yu, H. Dong, Z. Wang, W. Ren and F. E. Alsaadi, Design of non-fragile state estimators for discrete time-delayed neural networks with parameter uncertainties, Neurocomputing, vol. 182, pp. 18-24, 2016.

[30] N. Zeng, Z. Wang, Y. Li, M. Du and X. Liu, Identification of nonlinear lateral flow immunoassay state-space models via particle filter approach, IEEE Transactions on Nanotechnology, vol. 11, no. 2, pp. 321-327, 2012.

[31] N. Zeng, Z. Wang, Y. Li, M. Du and X. Liu, A hybrid EKF and switching PSO algorithm for joint state and parameter estimation of lateral flow immunoassay models, IEEE/ACM Transactions on Computational Biology and Bioinformatics, vol. 9, no. 2, pp. 321-329, 2012.

[32] N. Zeng, Z. Wang, Y. Li, M. Du and X. Liu, Inference of nonlinear state-space models for sandwich-type lateral flow immunoassay using extended Kalman filtering, IEEE Transactions on Biomedical Engineering, vol. 58, no. 7, pp. 1959-1966, 2011.

[33] N. Zeng, Z. Wang, Y. Li, M. Du, J. Cao and X. Liu, Time series modeling of nano-gold immunochromatographic assay via expectation maximization algorithm. IEEE Trans. Biomedical Engineering, vol. 60, no. 12, pp. 3418-3424, 2013.

[34] N. Zeng, Y.S. Hung, Y. Li, M. Du. A novel switching local evolutionary PSO for quantitative analysis of lateral flow immunoassay, Expert Systems with Application, vol. 41, no. 4, pp. 1708-1715, 2014.

[35] N. Zeng, Z. Wang, B. Zineddin, Y. Li, M. Du, L. Xiao, X. Liu, and T. Young, Image-based quantitative analysis of gold immunochromatographic strip via cellular neural network approach, IEEE Trans. Medical Imaging, vol. 33, no. 5, pp. 1129-1136, 2014.

[36] Z. Zhan, J. Zhang, Y. Li and H. Chung, Adaptive particle swarm optimization, IEEE Transactions on System, Man and Cybernetics-B, vol. 39, no. 6, pp. 1362-1381, 2009.

[37] G. Zhang, X. Wang, A. Zhi, Y. Bao, Y. Yang, M. Qu, J. Luo, Q. Li, J. Guo, Z. Wang, J. Yang, G. Xing, S. Chai, T. Shi and Q. Liu, Development of a lateral flow immunoassay strip for screening of sulfamonomethoxine residues, Food Addit Contam Part A, vol. 25, no. 4 pp. 413-423, 2008.

[38] J. Zhu, W. Chen, Y. Lu and G. Cheng, Development of an immunochromatographic assay for the rapid detection of bromoxynil in water, Environmental Pollution, vol. 156, no. 1, pp. 136-142, 2008. 